\title{
Seroprevalence of Anti-Toxoplasma IgG and IgM among Pregnant Women of Shush County, Southwest of Iran \\ Somayeh Fallahizadeh, ${ }^{1,2}$ Ali Jelowdar, ${ }^{1,3,}$, Forough Kazemi, ${ }^{1,2}$ and Bahman Cheraghian ${ }^{4}$
}

\author{
${ }^{1}$ Department of Parasitology, Faculty of Medicine, Ahvaz Jundishapur University of Medical Sciences, Ahvaz, IR Iran \\ ${ }^{2}$ Student Research Committee, Ahvaz Jundishapur University of Medical Sciences, Ahvaz, IR Iran \\ ${ }^{3}$ Health Research Institute, Infectious and Tropical Diseases Research Center, Ahvaz Jundishapur University of Medical Sciences, Ahvaz, IR Iran \\ ${ }^{4}$ Department of Statistics, Faculty of Health, Ahvaz Jundishapur University of Medical Sciences, Ahvaz, IR Iran \\ "Corresponding author: Ali Jelowdar, Department of Parasitology, Faculty of Medicine, Ahvaz Jundishapur University of Medical Sciences, Ahvaz, IR Iran. E-mail:
} jelowdar@gmail.com

Received 2017 February 05; Revised 2017 April 18; Accepted 2017 April 18.

\begin{abstract}
Background: Toxoplasma gondii is an obligate intracellular parasite that causes toxoplasmosis. T. gondii can cause serious pathological effects in the fetus of infected women.

Objectives: The aim of this study was to investigate the seroprevalence of anti-Toxoplasma IgG and IgM among pregnant women of Shush county, southwest of Iran.

Methods: This descriptive cross-sectional study was conducted in 276 pregnant women. IgG and IgM antibodies of $T$. gondii were measured by the Enzyme-linked immunosorbent assay (ELISA) test. SPSS version 21 statistical software was used for data analysis and Chi-square test for significant differences.

Results: Of 276 women, 85 (30.8\%) and 2 (0.7\%) were positive for IgG and IgM, respectively. Of 146 urban women, 43 (29.5\%) were positive for IgG and of 130 rural women, $42(32.3 \%)$ were positive for IgG. There was no significant difference between rural and urban women for IgG antibody ( $\mathrm{P}>0.05$ ). Also, 2 (1.4\%) out of 146 urban women were positive for IgM. All of the 130 rural women were negative for IgM. No significant difference was found between rural and urban women for IgM antibody $(\mathrm{P}>0.05)$.

Conclusions: The findings indicated there is a relatively high prevalence of $T$. gondii in the study pregnant women. Therefore, evaluation of T. gondii before marriage and pregnancy is important for women and the health authorities of Shush County must pay more attention to control and prevention of the transmission of the parasite to individuals.
\end{abstract}

Keywords: Seroprevalence, Pregnant women, Toxoplasmosis, Shush, Iran, Toxoplasma gondii

\section{Background}

Toxoplasma gondii (T. gondii) is an obligate intracellular protozoan belonging to phylum Apicomplexa (1). The parasite causes toxoplasmosis that can infect warm-blooded vertebrates such as humans, other mammals, and birds (2). The parasite exists in one-third of the world population, particularly in developing and low-income countries (3). The prevalence of toxoplasmosis widely differs between $30 \%$ and $60 \%$ in developed and developing communities, respectively (4). Toxoplasmosis happens mostly in tropical and subtropical regions. T. gondii needs two hosts for completion of its life cycle including the cat family as the definitive host and vertebrates as the intermediate host. The modes of transmission include the use of water contaminated with oocytes, raw or undercooked meat containing tissue cysts, and congenital transmission (5).

Immunocompromised persons and pregnant women are at high risk for toxoplasmosis (6). When women are in the first trimester of their pregnancy, the risk of transmission of the parasite to the fetus is less than $17 \%$ but the severity of symptoms is so high that it often leads to abortion. The risk of infection in the fetus in the second trimester of pregnancy is $25 \%$ and can lead to hydrocephalus, microcephaly, cerebral calcification, and chorioretinitis. The possibility of transmission to the fetus in the third trimester of pregnancy increases by 65\%. In this case, the disease severity has a late-onset in infants and usually is asymptomatic at birth and eye symptoms may appear years later (7). The prevalence of the parasite among pregnant women has been reported in America (31.7\%), Sudan (34.1\%), Austria (35\%), Senegal (40.2\%), Colombia (45.8\%), and Paris (83\%) (8). In addition, in various regions of Iran, for example, Tabriz, Khorram-Abad, and Kashan, the prevalence has been $21.6 \%$, 31\%, and 50.8\%, respectively (9-11). 


\section{Objectives}

T. gondii can cause serious pathological diseases in the fetus of infected women (1). Therefore, the aim of this study was to investigate the seroprevalence of anti-Toxoplasma IgG and IgM among pregnant women of Shush county, southwest of Iran.

\section{Methods}

\subsection{Study Area}

Shush is a county in Khuzestan province, southwest of Iran. The county has an area of 3.577 square kilometers and its population was reported 53.897 in 2006. Shush has a temperature of about $50^{\circ} \mathrm{C}$ in summer.

\subsection{Study Population}

This is a descriptive cross-sectional study. The study population comprised 276 pregnant women referring to Nezam Mafi hospital of Shush, to perform pregnancy tests, in 2016. The population was selected randomly. To participate in this research, an informed consent was taken from all subjects and the subjects were informed about the nature and purpose of the research (12).

\subsection{Sampling and Experiments}

$3 \mathrm{ml}$ blood samples were taken from all pregnant women referring to Nezam Mafi hospital of Shush county. After centrifuging, the isolated serum was placed in microtubes. The micro-tubes were maintained at $-20^{\circ} \mathrm{C}$ until the time of testing. Before testing, the samples were melted. Then, IgG and IgM antibodies of T. gondii were measured by Enzyme-linked immunosorbent assay (ELISA) test. In the ELISA method, IgG and IgM were measured by Indirect ELISA and Ab Capture ELISA methods, respectively (13).

\subsection{Statistical Analysis}

SPSS version 21 statistical software was used for data analysis and Chi-square test for significant differences. A $P$ value less than 0.05 was considered significant.

\section{Results}

The number of pregnant women who referred to Nezam Mafi hospital of Shush in 2016 was 276 including 146 (52.90\%) urban and 130 (47.10\%) rural women. In total, the mean age of women was 27.8 years. The mean age of urban women was 28.18 years with the minimum and maximum of 16 and 45 years, respectively. The mean age of rural women was 27.36 years with the minimum and maximum of 16 and 44 years, respectively.
Table 1 shows the results of T. gondii IgG antibody evaluation by ELISA in Pregnant women referring to Nezam Mafi hospital of Shush, southwest of Iran in 2016. Of 276 women, $85(30.8 \%)$ were positive for IgG. Of 146 urban women, 43 (29.5\%) were positive for IgG antibody and of 130 rural women, 42 (32.3\%) were positive for IgG. There was no significant difference between rural and urban women for $\operatorname{IgG}$ antibody $(\mathrm{P}>0.05)$. Table 2 shows the results of T.gondii IgM antibody evaluation by ELISA in pregnant women referring to Nezam Mafi Hospital of Shush, southwest of Iran in 2016. Of 276 women, 2 (0.7\%) were positive for IgM. Of 146 urban women, $2(1.4 \%)$ were positive for IgM antibody. All the 130 rural women were negative for IgM. No significant difference was found between rural and urban women for $\operatorname{IgM}$ antibody $(\mathrm{P}>0.05)$.

\section{Discussion}

Congenital toxoplasmosis usually occurs in the fetus of pregnant mothers infected with the parasite for the first time. Commonly, acquired toxoplasmosis is asymptomatic in pregnant women but in the fetus, congenital toxoplasmosis is one of the most important diseases that can cause hydrocephalus, microcephaly, cerebral calcification, and chorioretinitis $(2,7)$. Therefore, the current study was conducted to investigate the seroprevalence of antiToxoplasma IgG and IgM among Pregnant women of Shush county, southwest of Iran.

According to our results, of 276 pregnant women, 85 (30.8\%) and $2(0.7 \%)$ were positive for IgG and IgM, respectively. Of 276, 146 (52.90\%) and 130 (47.10\%) were urban and rural women, respectively. Of the 146 urban women, 43 (29.5\%) were positive for IgG and of the 130 rural women, 42 (32.3\%) were positive for IgG. There was no significant difference between rural and urban women for IgG antibody $(\mathrm{P}>0.05)$. Of the 146 urban women, 2 (1.4\%) were positive for IgM while all of the 130 rural women were negative for IgM. No significant difference was found between rural and urban women for IgM antibody $(\mathrm{P}>0.05)$. These results are consistent with those of previous research. In 2014, Firouz et al. in Chalus (north of Iran) studied the presence of IgG and IgM antibodies in 50 pregnant women by ELISA and Chemiluminescence (CLIA) and showed that 26 (52\%) and 28 (56\%) were positive for IgG by ELISA and CLIA, respectively. In both methods, the samples were not positive for IgM (13).

Toxoplasmosis happens mostly in tropical and subtropical regions (5); due to the high temperature of the county, this prevalence rate is reasonable. Similarly, in 2009, Fouladvand et al. in Bushehr city (southwest of Iran) studied the presence of IgG and IgM antibodies in 303 women of reproductive age and showed 71 and 10 of 
Table 1. Evaluation of T. gondii IgG Antibody by ELISA in Pregnant Women Referring to Nezam Mafi Hospital of Shush, Southwest of Iran in 2016

\begin{tabular}{|c|c|c|c|c|c|c|}
\hline & & & \multicolumn{2}{|c|}{ IgG } & \multirow[t]{2}{*}{ Total } & \multirow[t]{2}{*}{ Sig. } \\
\hline & & & Negative & Positive & & \\
\hline \multirow{3}{*}{ Area } & \multirow{2}{*}{ Urban } & Count & 103 & 43 & 146 & \\
\hline & & $\%$ within area & 70.5 & 29.5 & 100.0 & \multirow{3}{*}{0.608} \\
\hline & Rural & Count & 88 & 42 & 130 & \\
\hline \multirow[t]{2}{*}{ Total } & & Count & 191 & 85 & 276 & \\
\hline & & $\%$ within area & 69.2 & 30.8 & 100.0 & \\
\hline
\end{tabular}

Table 2. Evaluation of T. gondii IgM Antibody by ELISA in Pregnant Women Referring to Nezam Mafi Hospital of Shush, Southwest of Iran in 2016

\begin{tabular}{|c|c|c|c|c|c|c|}
\hline & & & \multicolumn{2}{|c|}{ IgM } & \multirow[t]{2}{*}{ Total } & \multirow[t]{2}{*}{ Sig. } \\
\hline & & & Negative & Positive & & \\
\hline \multirow{3}{*}{ Area } & \multirow{2}{*}{ Urban } & Count & 144 & 2 & 146 & \multirow{4}{*}{0.180} \\
\hline & & $\%$ within area & 98.6 & 1.4 & 100.0 & \\
\hline & Rural & Count & 130 & 0 & 130 & \\
\hline \multirow{2}{*}{ Total } & & Count & 274 & 2 & 276 & \\
\hline & & $\%$ within area & 99.3 & 0.7 & 100.0 & \\
\hline
\end{tabular}

them were positive for IgG and IgM, respectively (14). Also, in 2015, Saki et al. in Ahvaz studied the presence of IgG and IgM antibodies in 130 women with abortion and 130 women with normal delivery by ELISA method that $21.5 \%$ of the samples with normal delivery and $24.6 \%$ of the samples with abortion were positive for IgG antibodies. IgM antibody was detected in one woman who had aborted but not in women with normal childbirth (15). These results have also been achieved in other countries with the same climatic conditions. For example, in 2013, Tammam et al. in Egypt showed that of 76 pregnant women, 35 and 14 were positive for IgG and IgM, respectively (16). In addition, in 2015, Alghamdi et al. in Saudi Arabia showed that in 250 pregnant women, $32.5 \%$ and $6.4 \%$ of them were positive for IgG and IgM, respectively (17).

One of the risk factors for infection with the parasite is "contact with cat" and "place of residence". Oocysts shed in the feces of cats and they can survive in the environment under conditions such as moist soil, warm and humid weather for months and even years. Exposure to cats is easier in rural areas. Thus, living in rural areas increases the risk of infection by the parasite $(1,18)$.

The findings indicated there is a relatively high prevalence of $T$. gondii in pregnant women who referred to Nezam Mafi Hospital of Shush, southwest of Iran in 2016.
The results of this study can help plan programs to control and prevent the protozoan in women. One of the strengths of this research is the high sample size, but risk factors of infection with this parasite were not examined in women of Shush that was a limitation of the study. In addition, a standard questionnaire was not used for collecting data that adds to the study limitations.

In conclusion, the findings indicated that there is a relatively high prevalence of $T$. gondii in pregnant women referring to Nezam Mafi hospital of Shush, southwest of Iran in 2016. Therefore, the evaluation of $T$. gondii before marriage and pregnancy is important in women and the health authorities of Shush county must pay more attention to control and prevention of the transmission of the parasite to individuals.

\section{Acknowledgments}

We would like to acknowledge Ahvaz Jundishapur University of Medical Sciences and the study participants for their assistance.

\section{Footnotes}

Conflict of Interest Statement: None declared. 
Informed Consent: This was obtained from all participants included in the study.

\section{References}

1. Azadi T, Darabi M, Sayehmiri F, Sayehmiri K. Investigating the Prevalence of Toxoplasmosis in Iranian Pregnant Women: A Systematic Review and Meta-Analysis. Scientific J Ilam Uni Med Sci. 2017 515;25(1):14858. doi: 10.1016/j.actatropica.2016.03.003. [PubMed: 26952970].

2. Gharavi MJ, Rahnama N, Jahani MR. Seroepidemiological survey of Toxoplasma infections of mentally retarded children. Iran J Public Health. 2005;34(1):19-22.

3. Robert-Gangneux F, Darde ML. Epidemiology of and diagnostic strategies for toxoplasmosis. Clin Microbiol Rev. 2012;25(2):264-96. doi: 10.1128/CMR.05013-11. [PubMed: 22491772].

4. Daryani A, Sarvi S, Aarabi M, Mizani A, Ahmadpour E, Shokri A, et al Seroprevalence of Toxoplasma gondii in the Iranian general population: a systematic review and meta-analysis. Acta Trop. 2014;137:185-94. doi: 10.1016/j.actatropica.2014.05.015. [PubMed: 24887263].

5. Dubey JP. The history of Toxoplasma gondii-the first 100 years. J Eukaryot Microbiol. 200811 1;55(6):467-75. doi: 10.1111/j.15507408.2008.00345.x. [PubMed: 19120791].

6. Gharavi MJ, Jalali S, Khademvatan S, Heydari S. Detection of IgM and IgG anti-Toxoplasma antibodies in renal transplant recipients using ELFA, ELISA and ISAGA methods: comparison of pre- and posttransplantation status. Ann Trop Med Parasitol. 2011;105(5):367-71. doi: 10.1179/1364859411Y.0000000022. [PubMed: 21929878].

7. Benenson MW, Takafuji ET, Lemon SM, Greenup RL, Sulzer AJ. Oocyst-transmitted toxoplasmosis associated with ingestion of contaminated water. N Engl J Med. 1982;307(11):666-9. doi: 10.1056/NEJM198209093071107. [PubMed: 7110216].

8. Yad Yad MJ, Jomehzadeh N, Jafar Sameri M, Noorshahi N. Seroprevalence of Anti-Toxoplasma gondii Antibodies Among Pregnant Woman in South Khuzestan, Iran. Jundishapur J Microbiol. 2014;7(5). doi:10.5812/jjm.9998.
9. Arbabi M, Talari SA, Asmar M. Seroepidemiology of toxoplasmosis in Kashan, 1993. Feyz J Kashan Univ Med Sci.1997;1.

10. Cheraghipour K, Taherkhani H, Fallah M, Sheikhian A, Sardarian K, Rostami Nejad M, et al. Seroprevalence of toxoplasmosis in pregnant women admitted to the health centers of Khorram-Abad City, Iran. Avicenna J Clin Med. 2010;17(3):46-51.

11. Dalimiasl AAM. Sero-epidemiology of Toxoplasma Infection in PregnantWomen Referred to Al Zahra Hospital in Tabriz. HBI J 2012;20(3):55-62.

12. Kazemi F, Fallahizadeh S, Feiz Haddad MH. Seroepidemiological study of toxoplasmosis in hemodialysis patients of Ahvaz, Southwest of Iran. Int J BioMed Public Health. 2018;1(1):12-6. doi: 10.24200/ijbmph.2018.55340.

13. Firouz ZE, Kaboosi H, Nasiri AF, Tabatabaie SS, Golhasani-KF, Zaboli F.A comparative serological study of toxoplasmosis in pregnant women by CLIA and ELISA methods in Chalus City Iran. Iran Red Crescent Med J. 2014;16(4).

14. Fouladvand M, Barazesh A, Zandi K, Naeimi B, Tajbakhsh S. Seroepidemiological study of toxoplasmosis in childbearing age women in Bushehr City, south west of Iran in 2009. Afr J Biotechnol. 2010;9(36).

15. Saki J, Mohammadpour N, Moramezi F, Khademvatan S. Seroprevalence of Toxoplasma gondii in women who have aborted in comparison with the women with normal delivery in Ahvaz, southwest of Iran. The Scientific World Journal. 2015. doi: 10.1155/2015/764369. [PubMed: 25699288]

16. Tammam AE, Haridy MA, Abdellah AH, Ahmed SR, Fayed HM, Alsammani MA. Seroepidemiology of toxoplasma gondii infection in women with first trimester spontaneous miscarriage in qena governorate, egypt. J Clin Diagn Res. 2013;7(12):2870-3. doi: 10.7860/JCDR/2013/6480.3780. [PubMed: 24551661]

17. Alghamdi J, Elamin MH, Alhabib S. Prevalence and genotyping of Toxoplasma gondii among Saudi pregnant women in Saudi Arabia. Saudi Pharm J. 2016;24(6):645-51.

18. Rahimi MT, Daryani A, Sarvi S, Shokri A, Ahmadpour E, Teshnizi SH, et al. Cats and Toxoplasma gondii: A systematic review and meta-analysis in Iran. Onderstepoort J Vet Res. 2015;82(1). doi: 10.4102/ojvr.v82i1.823. 